\title{
Le Jésus de Voltaire
}

\section{Gwenaelle Boucher}

\section{(2) OpenEdition}

\section{Journals}

\section{Édition électronique}

URL : http://journals.openedition.org/studifrancesi/29853

DOI : $10.4000 /$ studifrancesi.29853

ISSN : 2427-5856

\section{Éditeur}

Rosenberg \& Sellier

\section{Édition imprimée}

Date de publication : 1 avril 2006

Pagination : 65-72

ISSN : 0039-2944

\section{Référence électronique}

Gwenaelle Boucher, «Le Jésus de Voltaire », Studi Francesi [En ligne], 148 (XLX | I) | 2006, mis en ligne le 30 novembre 2015, consulté le 19 avril 2021. URL : http://journals.openedition.org/studifrancesi/ 29853 ; DOI : https://doi.org/10.4000/studifrancesi.29853

\section{(c) (i) (9)}

Studi Francesi è distribuita con Licenza Creative Commons Attribuzione - Non commerciale - Non opere derivate 4.0 Internazionale. 


\section{Le Jésus de Voltaire}

\section{Si Jésus n'existait pas, faudrait-il l'inventer?}

Pour cet ancien élève des Jésuites qu'est Voltaire, la réponse à cette question parodiant un de ses vers célèbres ne fait aucun doute: c'est bien Dieu seulement et lui seul qu'il faudrait inventer ou réinventer, et non pas le fils de Dieu. Ainsi, Voltaire n'a de cesse qu'il conteste toujours la divinité de Jésus et s'insurge souvent contre l'image d'un Dieu fait homme, image sacrilège selon lui qui contrevient à sa grande idée de l'Etre suprême, créateur et horloger de l'univers. Aussi, s'il reconnaît la seule existence historique de Jésus, c'est pour la démythifier et souligner à l'envi ses déficiences et ses défaillances humaines justement. Dans le Traité sur la Tolérance, Voltaire souligne ainsi en 1763 toute l'humanité de Jésus qui puise sa force et sa grandeur jusque dans sa finitude et ses fragilités mêmes: «Si Jésus-Christ sembla craindre la mort, si l'angoisse qu'il ressentit fut si extrême qu'il en eut une sueur mêlée de sang, ce qui est le symptôme le plus violent et le plus rare, c'est qu'il daigna s'abaisser à toute la faiblesse du corps humain, qu'il avait revêtu. Son corps tremblait, et son âme était inébranlable; il nous apprenait que la vraie force, la vraie grandeur, consiste à supporter des maux sous lesquels notre nature succombe. Il y a un extrême courage à courir la mort en la redoutant ${ }^{\prime}$. C'est ainsi que Voltaire, achevant de nier toute transcendance divine à Jésus, le ramène sur terre pour le comparer longuement à Socrate qui, pour être philosophe, n'en est pas moins homme: «Si l'on ose comparer le sacré avec le profane, et un Dieu avec un homme, sa mort, humainement parlant, a beaucoup de rapport avec celle de Socrate. Le philosophe grec périt par la haine des sophistes, des prêtres, et des premiers du peuple: le législateur des chrétiens succomba sous la haine des scribes, des pharisiens, et des prêtres. Socrate pouvait éviter la mort, et il ne le voulut pas: Jésus-Christ s'offrit volontairement. Le philosophe pardonna non seulement à ses calomniateurs et à ses juges iniques, mais il les pria de traiter un jour ses enfants comme lui-même, s'ils étaient assez heureux pour mériter la haine comme lui: le législateur des chrétiens, infiniment supérieur, pria son père de pardonner à ses ennemis» ${ }^{2}$.

De même, dans l'article «Christianisme» du Dictionnaire philosophique, Voltaire s'appuie sur une vaste documentation historique pour présenter un démenti permanent de la divinité christique: «[Jésus] cacha à ses contemporains qu'il était fils de Dieu, éternellement engendré, consubstantiel à Dieu, et que le Saint-Esprit procédait du Père et du Fils. Il ne dit point que sa personne était composée de deux natures et de deux volontés; il voulut que ces grands mystères fussent annoncés aux hommes dans la suite des temps, par ceux qui seraient éclairés des lumières du Saint-Esprit. Tant qu'il vécut, il ne s'écarta en rien de la loi de ses pères; il ne montra aux hommes qu'un juste agréable à Dieu, persécuté par ses envieux, et condamné à mort par des magistrats prévenus» ${ }^{3}$. Toujours dans le but de renier non pas la personne de Jésus mais sa filiation sacrée, Voltaire estime nécessaire de consacrer en 1767 un autre article de dictionnaire à la «Divinité de Jésus» dans lequel il feint de contester les audaces du socinianisme anti-trinitaire: «Les sociniens, qui sont regardés comme les blasphémateurs, ne reconnaissent point la divinité de Jésus-Christ. Ils osent prétendre, avec les philosophes de l'antiquité, avec les juifs, les mahométans, et tant d'autres nations,

(1) Traité sur la tolérance, 1763, Ed. R. Pomeau, Paris, Garnier-Flammarion, 1989, p. 105.

(2) Ibid., p. 104-105.
(3) Dictionnaire philosophique, article «Christianisme», Ed. R. Pomeau, Paris, Garnier-Flammarion, 1964, p. 116-117. 
que l'idée d'un Dieu homme est monstrueuse, que la distance d'un Dieu à l'homme est infinie, et qu'il est impossible que l'Etre infini, immense, éternel, ait été reconnu dans un corps périssable. [...] Ils poussent l'audace jusqu'au point d'affirmer que les chrétiens passèrent trois siècles entiers à former peu à peu l'apothéose de Jésus, et qu'ils n'élevaient cet étonnant édifice qu'à l'exemple des païens, qui avaient divinisé des mortels» ${ }^{4}$.

Ainsi ces différents exemples sont tout à fait révélateurs de l'importance de ce qui apparaît comme un élément fondateur de toute la «religion» de Voltaire et comme un motif obsédant dans sa prose mais aussi dans ses vers qui, même s'ils sont peu prisés voire méprisés de nos jours, constituent toutefois une forme d'expression privilégiée pour un auteur se voulant avant tout poète en son temps. En n'omettant pas d'appuyer aussi notre analyse sur ce corpus versifié peu connu mais qui fourmille de formules et d'images significatives, nous pourrons nous demander si Voltaire n'a pas eu l'ambition de substituer ses propres discours à une parole biblique qui ne serait plus lue ni entendue.

Dès l'ode Le Vrai Dieu, le jeune poète de 21 ans seulement apostrophe très durement le fils de Dieu dont il offre une vision saisissante sur le chemin de croix:

Je vous vois, victime innocente,

Sous le faix d'une croix pesante,

Vous traîner jusqu'au triste lieu.

Tout est prêt pour le sacrifice:

Vous semblez, de vos maux complice,

Oublier que vous êtes Dieu?.

Même s'il reniera plus tard cette ode de jeunesse, Voltaire révèle ainsi une véritable interrogation sur la divinité et, peut-être par réaction contre l'enseignement de ses anciens maîtres jésuites, formule ses premières objections déistes contre l'Incarnation et la Rédemption.

Quelques années plus tard, l'épître $A$ Uranie constitue en 1722 une oeuvre majeure dans la pensée de Voltaire qui versifie alors une sorte de profession de foi antéchristique; le poète non seulement ne se dédit pas mais il développe même ses intuitions devenues désormais certitudes en achevant de fouler aux pieds le nom de Jésus, compagnon d'un peuple insensé et superstitieux dont il partage l'existence misérable:

Le fils de Dieu, Dieu même, oubliant sa puissance,

Se fait concitoyen de ce peuple odieux;

Dans les flancs d'une Juive il vient prendre naissance;

Il rampe sous sa mère; il souffre sous ses yeux

Les infirmités de l'enfance.

Longtemps vil ouvrier; le rabot à la main,

Ses beaux jours sont perdus dans ce lâche exercice;

Il prêche enfin trois ans le peuple iduméen,

Et périt du dernier supplice ${ }^{6}$.

Après avoir retracé en quelques vers blasphématoires le parcours historique de Jésus, l'auteur déploie cette vision indigne d'un faux Dieu voué au néant et dont la mort même est inutile, impuissante à sauver l'homme du péché:

(4) Dictionnaire philosophique, article «Divinité de Jésus», éd. cit. , p. 167.

(5) Ode Le Vrai Dieu, 1715, M.VIII.417. (M renvoie aux Oeuvres complètes de Voltaire, Ed. L. Moland, Paris, Garnier Frères, 1877-1885)

(6) Epître A Uranie, 1722, M.IX.360. 
Son sang du moins, le sang d'un Dieu mourant pour nous,

N'était-il pas d'un prix assez noble assez rare,

Pour suffire à parer les coups

Que l'enfer jaloux nous prépare?

Quoi! Dieu voulut mourir pour la salut de tous,

Et son trépas m'est inutile!

Achevant cette évocation terrible, Voltaire laisse entendre des accents indignés soulignant toutes les incohérences d'une divinité aveugle et cruelle qui n'aurait créé l'homme que pour l'avilir, le perdre et le détruire:

Quoi! l'on me vantera sa clémence facile,

Quand remontant au ciel il reprend son courroux,

Quand sa main nous replonge aux éternels abîmes,

Et quand par sa fureur effaçant ses bienfaits,

Ayant versé son sang pour expier nos crimes,

Il nous punit de ceux que nous n'avons point faits!

Ce Dieu poursuit encore, aveugle en sa colère,

Sur ses derniers enfants l'erreur d'un premier père;

Il en demande compte à cent peuple divers

Assis dans la nuit du mensonge;

Il punit du fond des enfers

L'ignorance invincible où lui-même il les plonge,

Lui qui veut éclairer et sauver l'univers! ${ }^{8}$

Et Voltaire de conclure en s'adressant aux peuples prétendument «infidèles» dont l'infidélité ne réside que dans leur seule étrangeté à cette fausse liturgie chrétienne:

Amérique, vastes contrées,

Peuples, que Dieu fit naître aux portes du soleil,

Vous, nations hyperborées,

Que l'erreur entretient dans un si long sommeil,

Serez-vous pour jamais à sa fureur livrées

Pour n'avoir pas su qu'autrefois

Dans un autre hémisphère, au fond de la Syrie,

Le fils d'un charpentier, enfanté de Marie,

Renié par Céphas, expira sur la croix?

Je ne reconnais point à cette indigne image

Le Dieu que je crois adorer;

Je croirais le déshonorer

Par une telle insulte et par un tel hommage?.

Ainsi, cette longue diatribe apparaît tout à fait représentative des engagements de Voltaire pour lequel l'image de Jésus-Christ symbolise les dogmes erronés du christianisme et en cristallise toutes les errances et les déviances sectaires; c'est en ce sens que Voltaire se détourne de la fallacieuse icône christique pour lancer une prière à l'Etre suprême:

Entends, Dieu que j'implore, entends du haut des cieux,

Une voix plaintive et sincère, 
Mon incrédulité ne doit pas te déplaire;

Mon coeur est ouvert à tes yeux:

L'insensé te blasphème, et moi je te révère:

Je ne suis pas chrétien mais c'est pour t'aimer mieux ${ }^{10}$.

Condamnée par Jean-Baptiste Rousseau à la vieillesse dévote ${ }^{11}$, cette téméraire pièce de vers apparaît ainsi décisive dans la formation philosophique de Voltaire qui la remaniera plusieurs fois, optant en 1772 pour le titre Le Pour et le Contre, c'est-àdire pour ou contre le christianisme. On ne s'étonnera pas que la balance penche sensiblement du côté du «Contre» qui s'étend sur quatre-vingt-cinq vers, au détriment $\mathrm{du}$ «Pour» qui n'occupe que quarante-et-un vers. On peut également remarquer que, dans cette sorte de premier bilan des arguments favorables et défavorables au christianisme, Voltaire préfère utiliser le vers dont la noblesse est adaptée à la gravité de la pensée pour mettre en ordre, clarifier une réflexion déiste grâce à la structure rigoureuse du poème dont l'alternance entre les octosyllabes et les alexandrins mime le constant balancement du «Pour» et du «Contre». La versification a donc des vertus et une rigueur propices aux tentatives de définition et à la formation d'une philosophie que Voltaire ne remettra plus en question puisqu'il se contentera simplement d'accroître le déséquilibre en faveur du «Contre».

Relayant les vers des poèmes, une anecdote, dûment attestée par les biographes, est tout à fait significative de cette volonté voltairienne à la fois démythifiante et démystifiante de la divinité christique: montrant une sorte d'émerveillement newtonien, Voltaire, dans une posture étrange et pour le moins inhabituelle chez lui, a pu en effet se prosterner devant la beauté de la nature, devant le soleil levant, qui semblent manifester la présence divine. «Je sors pour voir un peu le lever du soleil; cette Profession de foi d'un Vicaire Savoyard m'en a donné envie... voyons si Rousseau a dit vrai» ${ }^{12}$, déclare Voltaire, alors octogénaire, avant d'entreprendre d'escalader péniblement une petite montagne pour découvrir le magnifique spectacle de la nature à la pointe du jour: «... les roches pères [sic] du Jura, les sapins verts, se découpant sur le fond bleu du ciel dans les cimes, ou sur le jaune chaud et âpres des terres; au loin des prairies, des ruisseaux; les mille accidents de ce suave passage qui précède la Suisse, et l'annonce si bien, et enfin la vue se prolonge encore dans un horizon sans bornes, un immense cercle de feu empourprant tout le ciel. Devant cette sublimité de la nature, Voltaire est saisi de respect: il se découvre, se prosterne, et quand il peut parler, ses paroles sont un hymne! 'Je crois en Toi!' s'écria-t-il avec enthousiasme; puis décrivant avec son génie de poète, et la force de son âme, le tableau qui réveillait en lui tant d'émotions, au bout de chacune des véritables strophes qu'il improvisait, 'Dieu puissant! je crois!' répétait-il encore» ${ }^{13}$. Ainsi de nombreux témoignages concordent pour attester l'émotion, l'adoration même de Voltaire rencontrant l'évidence d'un Être suprême moins démontrée par les théories que révélée par l'univers ${ }^{14}$. Cependant,

(10) Ibid.

(11) J.-B. Rousseau s'offusque en effet de l'épître A Julie, première version de l'épître $A$ Uranie dont le texte ne nous est pas parvenu, et inspirera à l'admiration voltairienne déçue le huitain suivant: «Rousseau, sujet au camouflet, / Fut autrefois chassé, dit-on, / Du théâtre à coups de sifflet, / De Paris à coups de bâton: / Chez les Germains chacun sait comme / Il s'est garanti du fagot; / Il a fait enfin son dévot, / Ne pouvant faire l'honnête homme», Sur J.-B. Rousseau, 1736, M.X.517.

(12) Propos de Voltaire rapportés par Lord H.
BROUGHAM, Lives of Men of Letters of Science, who flourished in the time of George III, London, C. Knight, 1845, t. I, p. 142.

(13) Ibid

(14) Sur ces différents témoignages, voir R. Pomeau, La Religion de Voltaire, éd. cit. , p. 416-419. On retrouve dans les contes voltairiens la transcription de ces scènes de prosternation: «Parouba se mit à genoux, et dit: Les cieux annoncent Dieux», Histoire de Jenni, XXI, 1775, in Contes en vers et en prose, éd. cit. , t. II, p. 499. 
sans doute mû par quelque sursaut satanique, il ne peut s'empêcher de ponctuer par une sorte de profanation de la liturgie chrétienne sa prosternation véritable devant la beauté de la nature au lever du soleil, interrompant ses premiers émois enthousiastes. «... tout à coup, se relevant, il remit son chapeau, secoua la poussière de ses genoux, reprit sa figure plissée, et regardant le ciel comme il regardait quelquefois le marquis de Villette lorsque ce dernier disait une naïveté, il ajoute vivement: 'Quant à Monsieur le Fils et Madame sa Mère, c'est une autre affaire'» ${ }^{15}$. On ne peut que regretter de ne pas posséder les vers issus de cette première ferveur manifeste, mais force est de constater que l'impertinence malicieuse de ce diable d'homme désamorce irrémédiablement jusqu'aux moments extatiques au cours desquels Voltaire laisse libre cours à sa foi véritable pour dénoncer comme superstitieuse toute théorie de l'Incarnation.

Pour autant, il s'agit d'aller au-delà de ces railleries habituelles: il semble que Voltaire ne renie l'image de Jésus-Christ que pour mieux assumer lui-même un message christique auquel il ne cesse de rendre hommage, mais qui se trouve hélas avili, perverti par les fanatismes et les «infâmes» impostures cléricales. Ainsi Voltaire s'insurge contre les interprétations abusives de l'Evangile qui s'appuient sur tel passage biblique ou parole christique tirés de leur contexte pour légitimer les persécutions les plus meurtrières; évoquant le figuier maudit par Jésus-Christ, Voltaire écrit en ce sens dans le Traité sur la Tolérance: «On donne plusieurs explications différentes de ce miracle; mais y en a-t-il une seule qui puisse autoriser la persécution? Un figuier n'a pu donner des figues vers le commencement de mars, on l'a séché: est-ce une raison pour faire sécher nos frères de douleur dans tous les temps de l'année? Respectons dans l'Ecriture tout ce qui peut faire naître des difficultés dans nos esprits curieux et vains, mais n'en abusons pas pour être durs et implacables» ${ }^{16}$. Se fondant ensuite sur les épisodes de l'enfant prodigue accueilli chez lui à bras ouverts ou du samaritain charitable, Voltaire rappelle alors les discours et les actions de Jésus, toutes de bonté et de pardon, pour conclure sur cette formule: «Si vous voulez ressembler à JésusChrist, soyez martyrs, et non pas bourreaux $\gg^{17}$. Mettant en scène celui qu'il appelle «l'Homme-Dieu» dans le septième Discours en vers sur l'bomme, Voltaire paraît en effet faire siennes les paroles christiques puisqu'il ne cesse d'en appeler toujours à l'amour, l'amitié et la fraternité pour la conciliation de tous les hommes:

Quand l'ennemi divin des scribes et des prêtres

Chez Pilate autrefois fut traîné par des traîtres,

De cet air insolent qu'on nomme dignité,

Le Romain demanda, «Qu'est-ce que la vérité?»

L'Homme-Dieu, qui pouvait l'instruire ou le confondre,

A ce juge orgueilleux dédaigna de répondre:

Son silence éloquent disait assez à tous

Que ce vrai tant cherché ne fut point fait pour nous.

Mais lorsque, pénétré d'une ardeur ingénue,

Un simple citoyen l'aborda dans la rue,

Et que, disciple sage, il prétendit savoir

Quel est l'état de l'homme, et quel est son devoir;

Sur ce grand intérêt, sur ce point qui nous touche,

Celui qui savait tout ouvrit alors la bouche;

Et dictant d'un seul mot ses décrets solennels:

«Aimez Dieu, lui dit-il, mais aimez les mortels».

Voilà l'homme et sa loi, c'est assez: le ciel même

A daigné tout nous dire en ordonnant qu'on aime ${ }^{18}$.

(15) Lord H. Brougham, op. cit. , t. I, p. 142.

(16) Traité sur la Tolérance, éd. cit. , p. 103.
(17) Ibid., p. 107.

(18) Septième Discours en vers sur l'bomme, 
Ainsi, qu'il s'agisse de «soulager son frère» ou de «tirer son ami du sein de la misère ${ }^{19}$, les vers voltairiens martèlent sans fin les premiers préceptes divins pour dénoncer tout manquement à la règle de miséricorde:

Enfants ingrats d'un même père,

Si vous prétendez le servir,

$\mathrm{Si}$ vous aspirez à lui plaire,

Est-ce à force de vous haïr?

Est-ce en déchirant l'héritage

Qu'un père si tendre et si sage

Du haut des cieux nous a transmis?

L'amour était votre partage

Cruels! auriez-vous plus de rage,

Si vous étiez nés ennemis? ? $^{20}$

«Très sots enfants de Dieu, chérissez-vous en frères, / Et ne vous mordez plus pour d'absurdes chimères ${ }^{21}$ : réclamant sans cesse la paix des coeurs, Voltaire, dont on sait toute l'importance qu'il attache à cette forme noble de la relation affective qu'est l'amitié à laquelle il dédie un Temple, érige alors en modèle la bonté d'une âme simple et douce:

Un ignorant, qui de son frère

Soulage en secret la misère,

Est mon exemple et mon docteur;

Et l'esprit hautain qui dispute,

Qui condamne, qui persécute,

N'est qu'un détestable imposteur ${ }^{22}$.

C'est en ce sens que cette humanité d'un Jésus-Christ décidément humain trop humain semble finalement moins faire l'objet d'une condamnation que d'une revendication voltairienne: contenant ces exhortations inlassables à l'amitié, la fraternité ou la pitiée ${ }^{23}$, les appels à l'humanité sont tout à fait représentatifs de l'oeuvre de Voltaire, et notamment de son oeuvre poétique qui apparaît moins comme le langage des dieux que comme la langue des hommes. Au centre de la sensibilité voltairienne $e^{24}$, l'homme participe du divin ${ }^{25}$ et se fait, plus que Dieu même, l'objet de toutes les attentions et des voeux voltairiens les plus pieux:

1738-1742, Ed. H. T. Mason, Oxford, Voltaire Foundation, 1991, t. 17, p. 525. Mêmes accents dans les Lettres philosophiques: «Il faut aimer, et très tendrement, les créatures; il faut aimer sa patrie, se femme, son père, ses enfants; et il faut si bien les aimer que Dieu nous les fait les aimer malgré nous. Les principes contraires ne sont propres qu'à faire des barbares raisonneurs», Lettres philosophiques, XXV, in Mélanges, Ed. J. Van den Heuvel, Paris, Gallimard, Pléiade, 1961, p. 112.

(19) Septième Discours en vers sur l'bomme, éd. cit. , t. 17, p. 525-526.

(20) Variantes de l'ode Sur le fanatisme, 1732, M.VIII.432.

(21) Épître $A$ l'auteur du livre des 'Trois imposteurs', 1769, M.X.404.

(22) Ode Sur le fanatisme, 1732, M.VIII.430.

(23) «Ce n'est qu'aux malheureux que vous devez vos soins», Oedipe, II, 3, 1719, M.II.76.

(24) «L'homme est vraiment le centre de cette sensibilité, l'homme avec son histoire, ses progrès, sa civilisation, son idéal moral coupé de tout lien suspect avec les formes confuses de l'inconnu», R. Naves, Le Goût de Voltaire, 1938, réédition Genève, Slatkine Reprints, 1967, p. 496.

(25) Sur la religion posée à partir de l'homme, pour un homme qui n'est plus humilié par Dieu mais participe de l'infini, voir P. BÉNICHOU, Le Sacre de l'écrivain (1750-1830), réédition Paris, Gallimard, 1996, p. 31-33 notamment. «Le rejet du Péché originel est au fond de la foi nouvelle: en même temps, et par un même mouvement, répudiation rationnelle d'un mythe et protestation d'une sensibilité qui se proclame innocente et revendique le bonheur. Tel est l'Evangile de l'Homme de Lettres, la bonne nouvelle qu'il annonce à son siècle: la gloire de Dieu n'humilie pas l'homme; mieux, elle réside en lui. (...) Le credo que l'Homme de Lettres annonce est une magnification de l'humanité; c'est ce credo qui fonde son sacerdoce», Ibid., p. 32. 
Enfin, grâce en nos jours à la philosophie,

Qui de l'Europe au moins éclaire une partie,

Les mortels, plus instruits, en sont moins inhumains;

Le fer est émoussé, les bûchers sont éteints ${ }^{26}$.

«Rien d'humain ne lui est étranger» ${ }^{27}$, écrit Paul Valéry à propos de Voltaire qui versifie contre la destruction de l'homme $e^{28}$, et, s'adressant au roi de Danemark qui a accordé la liberté de la presse dans son pays, affirme parler «au nom du genre humain»:

Je me jette à tes pieds, au nom du genre humain.

Il parle par ma voix, il bénit ta clémence;

Tu rends ses droits à l'homme, et tu permets qu'on pense.

Sermons, romans, physique, ode, histoire, opéra,

Chacun peut tout écrire, et siffle qui voudra! ${ }^{29}$

En ce sens, Voltaire est ce nouveau grand prêtre d'une nouvelle Eglise philosophique qui voudrait redonner force et vie à un catéchisme constellé de préceptes raisonnables et accessibles. Loin qu'il ne soit l'Antéchrist, ne se fait-il pas ainsi le véritable fils de Dieu, lui qui oppose à la tyrannie divine de Louis Racine l'image d'un Dieu paternel:

Si ton style me plait, ton Dieu n'est pas le mien:

Tu m'en fais un tyran; je veux qu'il soit un père;

Ton hommage est forcé, mon culte est volontaire;

Mieux que toi de son sang je reconnais le prix:

Tu le sers en esclave, et je l'adore en fils ${ }^{30}$.

En prose et en vers, Voltaire n'a en effet de cesse qu'il souligne et signale cette filiation divine, en invoquant toujours un Dieu aimant et bienfaisant:

Viens, pénètre avec moi d'un pas respectueux,

Les profondeurs du sanctuaire

Du Dieu qu'on nous annonce, et qu'on cache à nos yeux.

Je veux aimer ce Dieu, je cherche en lui mon père,

On me montre un tyran que nous devons haïr ${ }^{31}$

C'est dans cette perspective que, au risque de faire frémir les antivoltairiens, quelques voix, et non des moindres, se sont élevées pour désigner la dimension christique de Voltaire, ce «Don Quichotte des malheureux $»^{32}$ dont le corps souffrant se couvre de stigmates à chaque anniversaire de la funeste Saint-Barthélemy: «Voltaire est celui qui souffre, écrit Jules Michelet, celui qui a pris pour lui toutes les douleurs des hommes, qui ressent, poursuit toute iniquité. Tout ce que le fanatisme et la tyrannie ont jamais fait de mal au monde, c'est à Voltaire qu'ils l'ont fait. Martyr, victime universelle, c'est lui qu'on égorgea à la Saint-Barthélemy, lui qu'on enterra aux ruines du Nouveau Monde, lui qu'on brûla à Séville, lui que le parlement de Toulouse roua

(26) Poème sur la loi naturelle, Troisième partie, in Mélanges, éd. cit. , p. 283.

(27) P. VAlÉRY, Variété, in Oeuvres, Paris, Gallimard, Pléiade, 1957, t. I, p. 522.

(28) «Vous qui vous élevez contre l'humanité / (...) Vous voulez changer l'homme, et vous le détruisez», Cinquième Discours en vers sur l'bomme,
1738-1742, éd. cit. , t. 17, p. 510.

(29) Épître Au roi de Danemark, 1771, M.X.422.

(30) A M. Louis Racine, 1722, M.X.479.

(31) Epître A Uranie, 1722, M.IX.359.

(32) «Je suis le Don Quichotte des malheureux», Lettre à Richelieu, 18 septembre 1769, D15903. 
avec Calas» ${ }^{33}$. En effet, celui que René Pomeau décrit comme un «convulsionnaire» ${ }^{34}$, est littéralement déchiré par le souvenir des décapités, anéanti par une fièvre pathologique le jour anniversaire du massacre des protestants: en proie aux cauchemars les plus atroces, il éprouve dans sa chair la souffrance des suppliciés ${ }^{35}$. C'est ainsi que, dans son discours prononcé lors du centenaire de la mort de Voltaire le 30 mai 1878, Victor Hugo n'hésite pas à opérer un rapprochement explicite entre Jésus et ce combattant du bien et de la bienfaisance dont les idées sont à ce point vécues qu'elles hantent véritablement son âme et son esprit de monstrueuses allégories et de figures affreuses: «Combattre le pharisaïsme, démasquer l'imposture, terrasser les tyrannies, les usurpations, les mensonges, les superstitions, démolir le temple, quitte à le rebâtir, c'est-à-dire à remplacer le faux par le vrai, attaquer la magistrature féroce, attaquer le sacerdoce sanguinaire, prendre un fouet et chasser les vendeurs du sanctuaire, réclamer l'héritage des déshérités, protéger les faibles, les pauvres, les souffrants, les accablés, lutter pour les persécutés et pour les opprimés; c'est la guerre de Jésus-Christ. Et quel est l'homme qui fait cette guerre? c'est Voltaire. L'oeuvre évangélique a pour complément l'oeuvre philosophique; l'esprit de mansuétude a commencé, l'esprit de tolérance a continué; disons-le avec un sentiment de respect profond, Jésus a pleuré, Voltaire a souri; c'est de cette larme divine et de ce sourire humain qu'est faite la douceur de la civilisation actuelle» ${ }^{36}$. Et, prévenant les cris horrifiés des esprits soi-disant philosophes devant cette comparaison inédite et pourtant évidente, Victor Hugo d'ajouter en note sur son manuscrit: «Jésus. Voltaire. Le rapprochement de ces deux noms ne peut étonner que les esprits superficiels $\rangle^{37}$.

GWENAELLE BOUCHER

(33) J. MicheLET, Histoire de la Révolution française, Introduction, in Oewvres complètes, Ed. P. VialLaneix, 1970, t. I, p. 56.

(34) «Il est, à sa façon, 'convulsionnaire', comme son frère. Le père Arouet disait: 'J'ai pour fils deux fous, l'un en prose, l'autre en vers'», R. Pomeau, Voltaire, Paris, Seuil, Points, 1989, p. 37.

(35) Certes, Voltaire assimile peut-être rapidement la passion religieuse à la passion criminelle, confondant systématiquement ferveur et fureur, mais à ses détracteurs, on peut opposer les réalités atroces des persécutions commises au nom de la religion au XVIIIe siècle: «Le siècle des philosophes était aussi l'époque où s'épanouissait la plus grossière superstition, et il y avait de quoi raviver les craintes d'un Voltaire dans le spectacle des convulsionnaires jansénistes criant 'Il faut du sang!' autour de la tombe du diacre Pâris», R. S. RidgWay, La Propagande philosophique dans les tragédies de Voltaire, in SVEC, XV, Genève, 1961, p. 131.

(36) V. Hugo, Discours prononcé lors du centenaire de la mort de Voltaire le 30 mai 1878, in Oeuvres complètes, Paris, Le Seuil, 1962, t. 15, p. 1431-1432.

(37) Ibid., p. 1432. 